\title{
The Affective Storyteller: Using Character Emotion to Influence Narrative Generation
}

\author{
Frank Kaptein and Joost Broekens \\ TU Delft, Interactive Intelligence, \\ Delft, The Netherlands \\ http://ii.tudelft.nl/
}

\begin{abstract}
We present the Affective Storyteller, a narrative generation framework that combines storytelling and emotion. With this framework we propose to address two main challenges in narrative generation: customization, and, reduced calculation time. Our solution is based on the fact that narrative generation in the Affective Storyteller is influenced by an analysis of the emotional patterns of the synthetic characters in the stories. These emotions are simulated using GAMYGDALA.
\end{abstract}

Keywords: Narrative generation, storytelling, emotion

\section{Introduction}

A story is the presentation of a series of logically and chronologically related events that are caused or experienced by actors [1]. Storytelling is a powerful tool to influentially communicate information $[5,11]$ in fields including entertainment, education, health care, advertising, training and argumentation [10]. Narratologists define a differentiation between the generation of the story events and the manner in which these are presented [9], it is referred to as fabula and presentation [1]. Fabula is the chronological order of all events that occur in the story. It can be presented in different ways. Examples are: speech, writing and movies.

Narrative generation is about the aim to have computers automatically generate stories (for review see [4]). Computers are capable of generating multiple story lines each with the same message but told differently. This improves replay value (retelling the story to the same user) and personalizing the story to a particular user [9]. By using simulated emotions of virtual characters, this research proposes a method to improve the generation efficiency and to enable customization of the story.

\subsection{Emotions in Synthetic Storytelling}

Emotion can be used to measure the user and steer the story based on those emotions, or to model the emotions of the virtual characters [3,14]. We focus on 
this second approach. The success of a story is dependent on the understandability and believability of its characters [8], characters need emotions to be believable [2]. The work in [7] tries to identify how affective characters should be configured. This approach is also seen in the way the virtual storyteller uses emotional modeling $[12,13]$.

\section{Challenges in narrative generation}

There are two challenges that we consider here. The first is efficiency: computation time grows exponentially in the size of the story domain. Heuristics are needed that can reject story paths before completely calculating them. The second is customization: how to make different versions of the same story, for example to facilitate replay or personal style of the reader. In this research we propose to use the characters' simulated emotions to address these challenges.

\section{Approach}

We have developed the Affective Storyteller, a domain independent system that uses GAMYGDALA [6] to generate character emotions. These emotions are used to improve on the previously mentioned challenges.

\subsection{Customization of story affect}

The narrative generation problem in the Affective storyteller starts with the user defining a domain. The Affective storyteller then calculates all possible event sequences that lead to the defined finishing state of the story. These event sequences differ in storyline, and character emotions. The problem we address here is how to find the subset of event sequences that fit particular affective preferences.

In every event sequence the GAMYGDALA-calculated emotions for the different characters are measured and logged. These are then used to sort the stories. The user is presented an emotion panel that shows all occurring emotions in the characters during the story. The panel makes it possible to change the importance of these emotions. When a user for example increases the importance for Joy, then the Affective storyteller will search for stories that have more joy occurring in them. Currently, a user can choose these values for the first half and the second half separately, but we anticipate extending this with regular expression-like functionality that can be used to filter the story.

The Affective storyteller uses GAMYGDALA to generate 16 different emotions after both the first and second half of the story, being a total of 32 measurements for each event sequence. The user chooses for every emotion, for both halfs of the story, how important this occurrence is to him on a scale from 0 to 1 . This again results in a total of 32 numbers. We use a normalized dot product of these two vectors as a value function for the different event sequences. In equation 1 EI stands for emotion intensity, EC is the emotion congruency as defined 
by the user, and Gain is a constant set by the user defining how aggressive the intensity dampening is.

$$
\operatorname{Value}(\operatorname{Seq})=\sum_{i=1}^{32}\left\{\left(\frac{E I_{i} * \text { gain }}{E I_{i} * \text { gain }+1}\right) * E C_{i}\right\} .
$$

When the fabula generation has been affectively customized, the best matching story is transformed in a written text communicating the events with their main affective consequences.

\subsection{Efficiency}

The fabula generation method as explained in section 3.1 starts by calculating all possible story-paths and then sorts these based on the similarity of their emotion pattern and the user preference. Addressing the performance problem (section 2), we show as a proof of concept heuristic( in our case, and as an example, based on conflict between the characters) enhances efficiency. Conflict between two characters means they perform actions that are undesirable for the other. GAMYGDALA generates anger for these type of actions, which means that stories with insufficient anger should be pruned.

In table 3.2 the calculation time of the pruning algorithm is compared with that of the complete breadth first search. The algorithms are tested on an example domain where idle actions are incrementally added. These idle actions can occur once in the story but at every moment of the story. Initially the breadth first search is quicker because it does not have the overhead of calculating the emotions of the characters. However, because the number of possible paths grows exponentially and this pruning heuristic decreases the exponent, the computation time of the emotionally-pruned algorithm quickly outperforms the classic breadth-first algorithm.

\begin{tabular}{|l|c|c|c|c|}
\hline & Initial domain & 1 Idle action & 2 Idle action & 3 Idle action \\
\hline Prune on anger & $2,27 \mathrm{sec}$ & $5,31 \mathrm{sec}$ & $8,57 \mathrm{sec}$ & $12,25 \mathrm{sec}$ \\
\hline Breadthfirst without emotions & $0,04 \mathrm{sec}$ & $0,27 \mathrm{sec}$ & $7,26 \mathrm{sec}$ & $26 \mathrm{~min} ; 31 \mathrm{sec}$ \\
\hline
\end{tabular}

Table 1. Time of calculation. Method 1 prunes on anger to reduce the search space. Method 2 saves time by skipping emotion calculations.

\section{Discussion \& future work}

We have proposed a framework that affectively models the story-characters to customize stories and improve the efficiency of their generation. These emotions can be used to filter the set of generated stories, either during or after generation of the set. In both cases, it is important to be able to correctly represent 
the emotional patterns one is looking for in a story. Now we used a simple vector-based filter method, but we anticipate using a regular expression type of filtering so that more complex patterns can be found, which is especially useful in longer stories. Further, we have shown that it is possible to optimize the story generation process by pruning on emotional filters. When the domain becomes more complex, this will dramatically reduce processing time. Although there is much more work to be done on the exploration of emotion patterns in stories, in particular verification of the perceived story quality, we conclude based on our current work that emotion simulation is a promising direction to address planning complexity and story customization.

\section{References}

1. Bal, M., Van Boheemen, C.: Narratology: Introduction to the theory of narrative. University of Toronto Press (2009)

2. Bates, J., et al.: The role of emotion in believable agents. Communications of the ACM 37(7), 122-125 (1994)

3. Cavazza, M., Pizzi, D., Charles, F., Vogt, T., André, E.: Emotional input for character-based interactive storytelling. In: Proceedings of The 8th International Conference on Autonomous Agents and Multiagent Systems-Volume 1. pp. 313320. International Foundation for Autonomous Agents and Multiagent Systems (2009)

4. Gervás, P.: Computational approaches to storytelling and creativity. AI Magazine 30(3), 49-62 (2009)

5. Mar, R.a., Oatley, K., Djikic, M., Mullin, J.: Emotion and narrative fiction: Interactive influences before, during, and after reading. Cognition \& emotion 25(5), 818-33 (Aug 2011), http://www.ncbi.nlm.nih.gov/pubmed/21824023

6. Popescu, A., Broekens, J., van Someren, M.: GAMYGDALA: An Emotion Engine for Games. IEEE Transactions on Affective Computing 5(1), 32-44 (Jan 2014), http://ieeexplore. ieee. org/lpdocs/epic03/wrapper. htm?arnumber $=6636311$

7. Rank, S., Hoffmann, S., Struck, H.G., Spierling, U., Petta, P.: Creativity in configuring affective agents for interactive storytelling. In: International conference on computational creativity. p. 165 (2012)

8. Riedl, M.O., Young, R.M.: An objective character believability evaluation procedure for multi-agent story generation systems. In: Intelligent Virtual Agents. pp. 278-291. Springer (2005)

9. Riedl, M.O., Young, R.M.: Narrative planning: Balancing plot and character. Journal of Artificial Intelligence Research 39, 217-268 (2010)

10. Riedl, M.O., Bulitko, V.: Interactive narrative: An intelligent systems approach. AI Magazine 34(1), 67 (2012)

11. Simmons, A.: The story factor: Secrets of influence from the art of storytelling. Basic books (2006)

12. Swartjes, I., Theune, M.: A fabula model for emergent narrative. In: Technologies for Interactive Digital Storytelling and Entertainment, pp. 49-60. Springer (2006)

13. Theune, M., Rensen, S., op den Akker, R., Heylen, D., Nijholt, A.: Emotional characters for automatic plot creation. In: Technologies for Interactive Digital Storytelling and Entertainment, pp. 95-100. Springer (2004)

14. Zhao, H.: Emotion in interactive storytelling. In: FDG. pp. 183-189 (2013) 